\title{
Grid-free Simulation of Radiative Transport in a Participating Medium
}

\author{
I. LAKKIS, A.F. GHONIEM \\ Department of Mechanical Engineering, Rm 3-339 \\ Massachusetts Institute of Technology \\ Cambridge, MA 02139, U.S.A. \\ ilakkis@mit.edu,ghoniem@mit.edu
}

\begin{abstract}
Radiative transport in a participating medium transfroms the energy equation into an integro-differential equation which must be solved if radiation is considered as an important mechanism of heat transfer in a convective-diffusive-reactive environment. In most cases, especially in combustion problems, the temperature gradients are so steep that the region where the radiative flux, which is proportional to the fourth power of temperature, is significant in a small subset of the entire flow domain. Especially when utilizing a grid-free Lagrangian scheme to perform the convective-diffusive-reactive simulations, it is desirable to develop a compatible scheme which does not require a grid to perform the computations of the radiative flux and its gradient (needed in the energy equation.) We have developed the discrete source method in which the radiative flux and irradiance are computed by summing over a collection of radiating elements distributed over the region of high temperature. In an axisymmetric domain, the irradiance and radiative flux, both are triple integrals of the radiation kernel over the elemental volume, are reduced to double integrals which are then approximated by single integrals that are evaluated semi-analytically. We demonstrate the accuracy of the scheme by comparing the results with direct numerical evaluation of the original integrals for cases relevant to combustion problems.
\end{abstract}

\section{Radiation in a Gray Non-Scattering Medium}

For a gray non-scattering medium, the specific intensity $I(\mathbf{r}, \hat{s})$, the energy transfer rate per unit area per unit solid angle, of a radiation field at the point $\mathbf{r}$ in the direction $\hat{s}$ is given by $[1]$

$$
I(\mathbf{r}, \hat{s})=I\left(\mathbf{r}_{\Sigma}^{\prime}\right) e^{-\int_{0}^{s} a\left(s^{\prime \prime}\right) d s^{\prime \prime}}+\int_{0}^{s} i_{b}\left(\mathbf{r}^{\prime}, \hat{s}\right) e^{-\int_{s^{\prime}}^{s} d s^{\prime \prime}} a\left(s^{\prime}\right) d s^{\prime}
$$

Article published by EDP Sciences and available at http://www.edpsciences.org/proc or http://dx.doi.org/10.1051/proc:1999022 where $a$ is the absorption coefficient. The black body intensity, $i_{b}$, is given by $i_{b}=\sigma T^{4} / \pi$, where $\sigma$ is the Stefan-Boltzmann constant (the refractive index of the medium is assumed to be unity). The source and target are defined by vectors $\mathbf{r}$ and $\mathbf{r}^{\prime}$ respectively, and $\mathbf{s}=s \hat{s}$ is the coordinate along the ray emanating from $\mathbf{r}_{\Sigma}^{\prime}$ at the boundary $(\Sigma)$ and passing through target $\mathbf{r}$. The outer integration in the second term of the right-hand side of Eq. (1) is along $\mathbf{s}$ from a point $s^{\prime}=0$ at the boundary to the target point $s^{\prime}=s$ inside the medium.

The total radiative heat flux vector is the intensity integrated over all solid angles $\Omega$

$$
\mathbf{q}(\mathbf{r})=\int_{4 \pi} I(\mathbf{r}, \hat{s}) \hat{s} d \Omega
$$

where the solid angle $d \Omega=d A^{\prime} /\left|\mathbf{r}-\mathbf{r}^{\prime}\right|^{2}$ and the unit vector $\hat{s}=\left(\mathbf{r}-\mathbf{r}^{\prime}\right) /\left|\mathbf{r}-\mathbf{r}^{\prime}\right|$.

The divergence of the heat flux, the negative of which is the radiation source term in the energy equation, is given by 


$$
\nabla \cdot \mathbf{q}(\mathbf{r})=a(\mathbf{r})\left(4 \pi i_{b}(\mathbf{r})-G(\mathbf{r})\right)
$$

where $G$, the irradiance, is given by

$$
G(\mathbf{r})=\int_{4 \pi} I(\mathbf{r}, \hat{s}) d \Omega
$$

Assuming that the incident intensity crossing the boundary is zero, the irradiance is expressed as [2]

$$
G(\mathbf{r})=\int_{V} a\left(\mathbf{r}^{\prime}\right) i_{b}\left(\mathbf{r}^{\prime}\right) \frac{e^{-\int_{s^{\prime}}^{s} a\left(s^{\prime \prime}\right) d s^{\prime \prime}}}{\left|\mathbf{r}-\mathbf{r}^{\prime}\right|^{2}} d V\left(\mathbf{r}^{\prime}\right)
$$

\section{The Discrete Source Method}

Our objective is to simulate radiative transport using a grid free scheme compatible with the vortex method (flow simulation) and the transport element method (combustion simulation). To meet this objective, we propose the Discrete Source Method. This method enables the calculation of the heat flux and its divergence, induced by a collection of computational elements that discretize the temperature field. The solution is presented in terms of a "Biot-Savart" summation over the computational elements decomposing the domain.

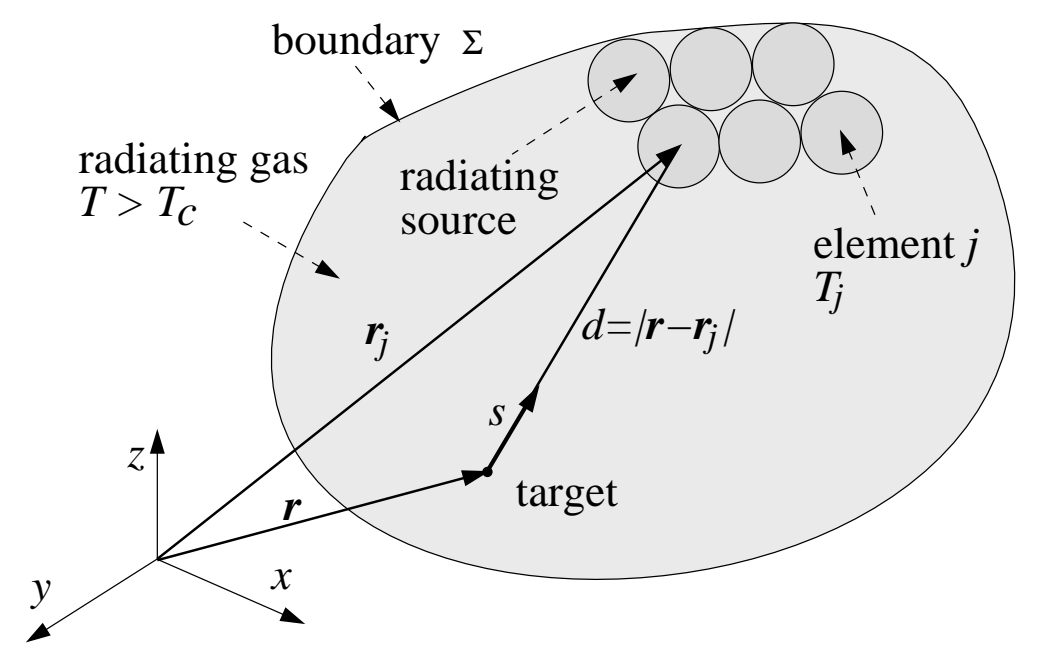

Figure 1: Decomposing the participating medium into computational elements.

If we select volume $V$ to be large enough such that it is surrounded by a cold medium, Fig. 1 , the incident intensity crossing the boundary can be neglected, and the irradiance is given by Eq. (5).

Decomposing volume $V$ into $N$ elemental volumes, and assuming that the temperature $T_{j}$ and absorption coefficient $a_{j}$ are uniform within each element, $j=1, N$, then the irradiance is expressed in the following discretized form

$$
G(\mathbf{r})=\sum_{j=1}^{N} G_{j}(\mathbf{r})
$$

where $G_{j}(\mathbf{r})$ is the irradiance induced by computational element $j$ :

$$
G_{j}(\mathbf{r}) \simeq a_{j} i_{b_{j}} \int_{V_{j}} \frac{e^{-\bar{a}_{j}\left|\mathbf{r}-\mathbf{r}^{\prime}\right|}}{\left|\mathbf{r}-\mathbf{r}^{\prime}\right|^{2}} d V\left(\mathbf{r}^{\prime}\right)
$$


where $\bar{a}_{j}$, defined as the "effective attenuation coefficient", is given by

$$
\bar{a}_{j}=\frac{\int_{0}^{\left|\mathbf{r}-\mathbf{r}_{j}\right|} a(s) d s}{\left|\mathbf{r}-\mathbf{r}_{j}\right|}
$$

The effective attenuation coefficient is obtained by assuming that $\left|\mathbf{r}-\mathbf{r}^{\prime}\right| \simeq\left|\mathbf{r}-\mathbf{r}_{j}\right|$ in evaluating the inner integral of Eq. (5). This approximation, justified by selecting small enough computational elements, simplifies computing the integral considerably. For a sourcetarget configuration, the effective attenuation coefficient is calculated once, according to Eq. (8), and is then used as a constant in the triple integral (7). Evaluation of $\bar{a}_{j}$ requires integration of the absorption coefficient along the line of sight between traget and source center. A simple way is to determine the absorption coefficient on a grid and evaluate $\bar{a}_{j}$ assuming a piecewise profile of $a$. The cost entails calculating the temperature on the grid and constructing a data structure suitable for finding the grid cells relevant to a certain line of sight.

\section{Solution for a Spherical Element}

Consider a spherical radiative source of radius $\delta$ and absorption coefficient $a$. The irradiance at distance $\rho$ from the center of the sphere is given by [3]

$$
\begin{gathered}
\frac{G(\rho \leq \delta)}{4 \pi i_{b}}=\frac{a}{\bar{a}}+\frac{a}{4 \rho}\left(\rho^{2}-\delta^{2}\right) \Delta E-\frac{1}{2} e^{-\bar{a} \delta}\left(\sinh (\bar{a} \rho)\left(\frac{a \delta}{\bar{a} \rho}+\frac{a}{\bar{a}^{2} \rho}\right)+\frac{a}{\bar{a}} \cosh (\bar{a} \rho)\right) \\
\frac{G(\rho \geq \delta)}{4 \pi i_{b}}=\frac{a}{4 \rho}\left(\rho^{2}-\delta^{2}\right) \Delta E+\frac{1}{2} e^{-\bar{a} \rho}\left(\sinh (\bar{a} \delta)\left(\frac{a}{\bar{a}}-\frac{a}{\bar{a}^{2} \rho}\right)+\frac{a \delta}{\bar{a} \rho} \cosh (\bar{a} \delta)\right)
\end{gathered}
$$

where $\Delta E=E i_{1}(\bar{a}(\rho+\delta))-E i_{1}(\bar{a}|\rho-\delta|), E i_{1}(x)=\int_{1}^{\infty} e^{-x t} t^{-1} d t$ is the exponential integral, and $\bar{a}=\int_{0}^{\rho} a(s) d s / \rho$. Of special interest is the "self-induced" irradiance, defined as the irradiance at the center of the sphere. The self-induced irradiance is given by

$$
\frac{G_{\text {self-sphere }}}{4 \pi i_{b}}=1-e^{-a \delta}
$$

\section{Solution for an Axisymmetric Element}

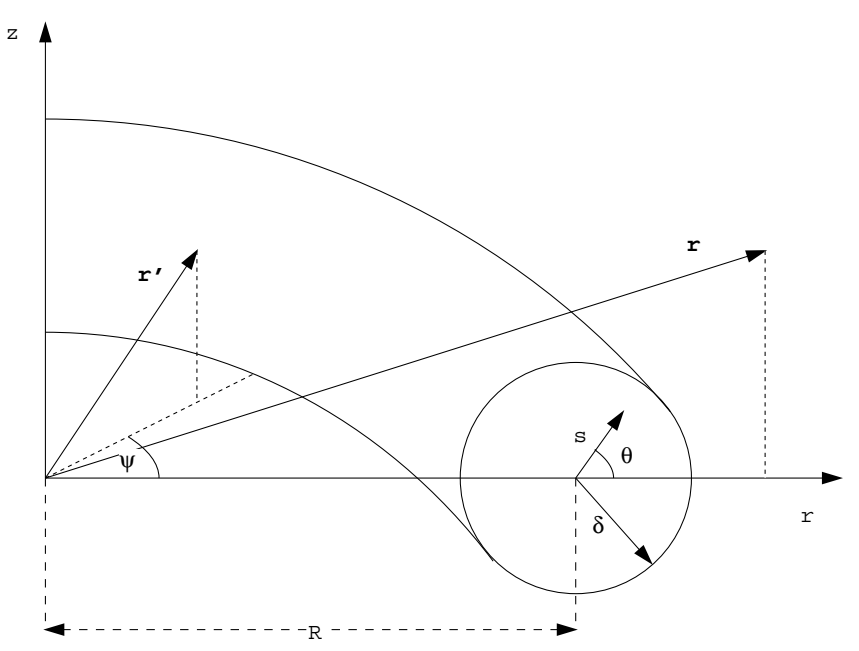

Figure 2: Ring in polar cylindrical coordinates. 
Consider a ring of radius $R$, cross-section radius $\delta$, blackbody intensity $i_{b}$, and absorption coefficient $a$, as shown in Fig. 2. In polar-cylindrical coordinates $(s, \theta, \psi)$, the irradiance at location $\mathbf{r}(r, 0, z)$ induced is

$$
G(\mathbf{r})=a i_{b} \int_{0}^{2 \pi} \int_{0}^{2 \pi} \int_{0}^{\delta} K_{G}\left(\mathbf{r}, \mathbf{r}^{\prime}\right) s(R+s \cos \theta) d s d \theta d \psi
$$

where

$$
K_{G}\left(\mathbf{r}, \mathbf{r}^{\prime}\right)=\frac{e^{-\bar{a} \sqrt{s^{2}-2 s(z \sin \theta-(R-r \cos \psi) \cos \theta)-2 r R \cos \psi+d^{2}}}}{s^{2}-2 s(z \sin \theta-(R-r \cos \psi) \cos \theta)-2 r R \cos \psi+d^{2}},
$$

$d^{2}=r^{2}+z^{2}+R^{2}$, and $\bar{a}=\int_{0}^{\left|\mathbf{r}-\mathbf{r}^{\prime}\right|} a(s) /\left|\mathbf{r}-\mathbf{r}^{\prime}\right| d s$

\subsection{Thin Ring Approximation}

For the case of a thin ring, characterized by $s / R \leq \delta / R \ll 1$, and if the effective attenuation coefficient is such that $\bar{a} \delta<1$ (optically-thin), an approximation is obtained by expanding the integrand of integral (12) in Taylor series in $s$ to second order. The irradiance is then approximated by

$$
G(\mathbf{r})=4 a i_{b} \int_{0}^{2 \pi} \int_{0}^{2 \pi} \int_{0}^{\delta}\left(\frac{e^{-\bar{a} \lambda \sqrt{1-\kappa^{2} \cos ^{2} \beta}}}{\lambda^{2}\left(1-\kappa^{2} \cos ^{2} \beta\right)} s R+O\left((\bar{a} s)^{2}\right)\right) d s d \theta d \beta
$$

where $\lambda^{2}=(r+R)^{2}+z^{2}, \kappa^{2}=4 r R / \lambda^{2}$, and $\psi=2 \beta$.

Integrating in $s$ and $\theta$ directions leads to

$$
G(\mathbf{r})=i_{b} \frac{4 \pi \delta^{2} R}{\lambda^{2}} f(\alpha, \kappa)+O\left((\bar{a} \delta)^{3}\right)
$$

where $\alpha=\bar{a} \lambda$ and

$$
f(\alpha, \kappa)=\int_{0}^{\pi / 2} \frac{e^{-\alpha \sqrt{1-\kappa^{2} \cos ^{2} \beta}}}{1-\kappa^{2} \cos ^{2} \beta} d \beta
$$

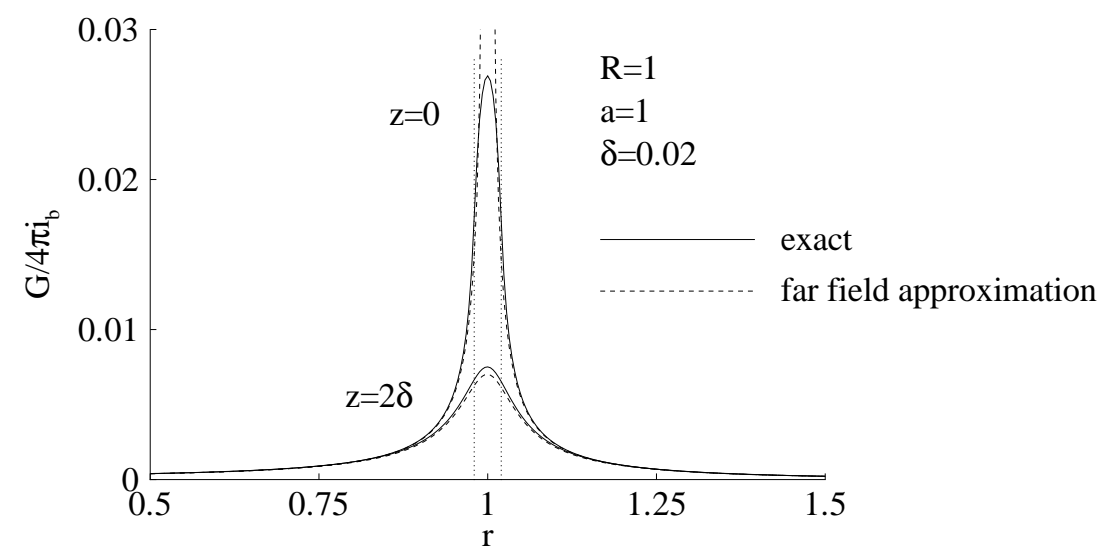

Figure 3: Irradiance due to a thin ring with small $a$

The thin ring approximation provides a "good" approximation of the heat flux and its divergence away from the core for values of $\bar{a}$ satisfying $\bar{a} \delta<1$. This is clearly observed in Fig. 3 , where radial profiles of the irradiance at two elevations are presented for a thin ring with 
the following parameters: $R=1, a=1, \delta=0.02$. Inside the core, however, this approximation diverges, and it is singular at the center of the core $(\kappa=1)$. In the following two sections, approximate solutions for $G$ for large values of $\bar{a}$ and/or inside the core are presented.

We note that desingularization of the integrand of Eq. (16) proved ineffective, especially for large values of the effective attenuation coefficient $\bar{a}$. The reason is that small perturbations in this integrand get magnified due to the "exponentiation effect" of $\bar{a}$. For example, consider the case where $a \delta=5$. Here, the kernel of $G$ is negligible at the edge of the core, and the sole contribution at the center of the element is due to the element itself. In this case, we require better than just killing the singularity for an accurate representation of the radiation field. The solution to this problem is presented in the next section.

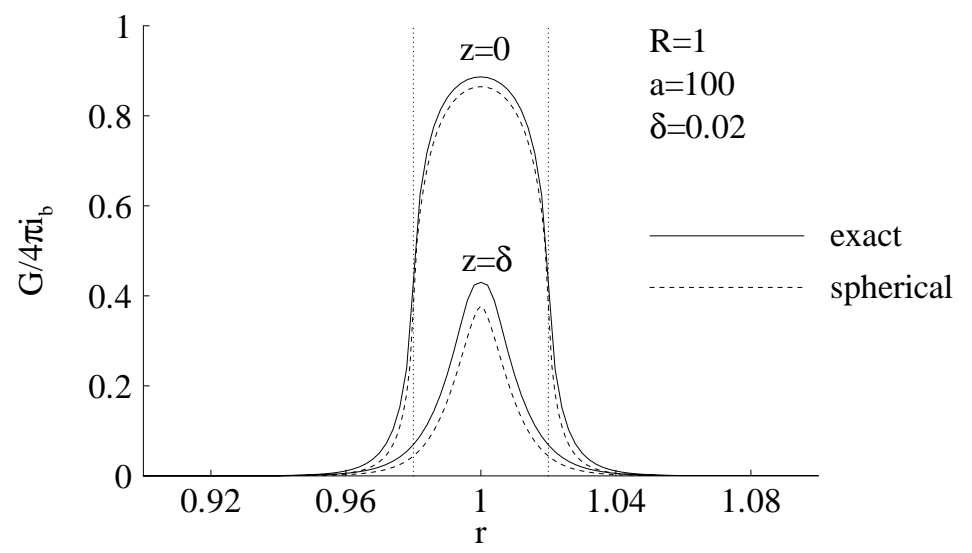

Figure 4: Ring and sphere solutions for large $a$

\section{Treatment of large absorption coefficient}

For large $\bar{a}$, the integral decays fast and the contribution of sources with $\bar{a} \delta \gg 1$ is in their immediate vicinity. Thus, it is convenient, under the condition $\bar{a} \delta>1$, to approximate $G$ by its value corresponding to a spherical element centered at $R$ with a radius $\delta$.

To show the usefulness of the sphere solution, Eqs. (9) and (10), for rings with $\bar{a} \delta>1$, radial profiles of irradiance due to a ring with $R=1, \delta=0.02, a=100$ are presented in Fig. 4 along with those obtained for a sphere located at $R=1$ and of radius $\delta=0.02$. The two profiles show good agreement.

\section{Solution inside the core}

The solution outside the core of an optically-thin computational ring has been presented in section 4.1. Further, an approximation of the solution for an optically-thick ring was proposed in the previous section. In this section, we propose an approximation inside the core of an optically-thin ring using a scaling of the spherical element solution to match the induced irradiance at the center of the element. Accrording to Eq. (12), the self induced irradiance is given by

$$
G_{\text {self }}=\frac{a}{\pi} \int_{0}^{\pi / 2} \int_{0}^{2 \pi} \int_{0}^{\delta} \frac{e^{-a \sqrt{s^{2}+4 \sin ^{2} \beta(s \cos \theta+1)}}}{s^{2}+4 \sin ^{2} \beta(s \cos \theta+1)} s(1+s \cos \theta) d s d \theta d \beta
$$

where $s, a$, and $\delta / R$, are non-dimensionalized by $R$, and $G_{\text {self }}=G(R, 0) /\left(4 \pi i_{b}\right)$. Integral (17) was calculated numerically for the ranges of $\delta \in[0.01,1]$ and $a \in[0,100]$. The solution 


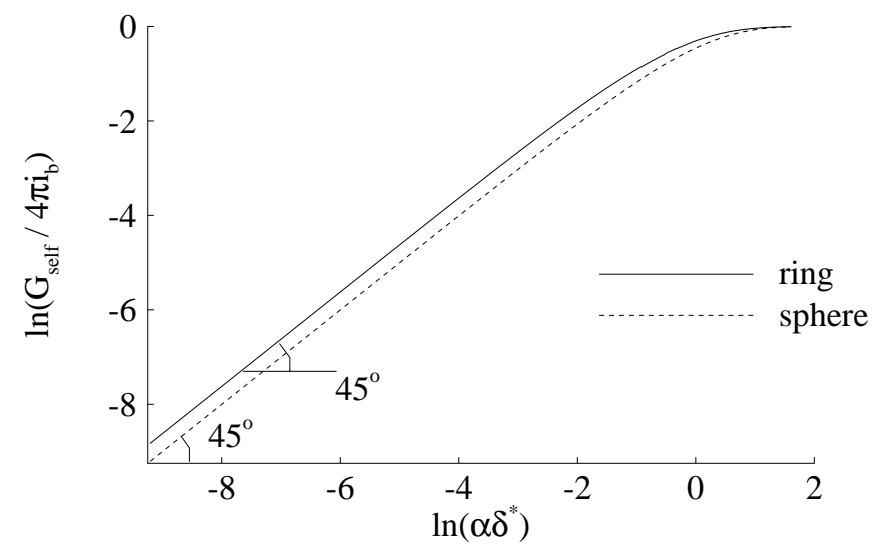

Figure 5: $G_{\text {self }}$ for both a ring and a sphere as a function of $a \delta$.

is presented in Fig. 5, showing that $\ln \left(G_{\text {self }}\right)$ is a linear function of $\ln (a \delta)$ for $\ln (a \delta) \leq-3$, while it approaches the optically-thick limit non-linearly for $\ln (a \delta)>-3$. In the same figure, the value for a spherical element is presented.

Knowing the value of the self induced irradiance, and observing that as $a \delta$ increases the solution for $G$ approaches that for a spherical element, the solutions for an optically-thick ring $(a \delta>1)$, or inside the core of an optically-thin ring $(a \delta<1)$ are approximated by the corresponding spherical element solution scaled by the ratio of the ring self-induced irradiance to the sphere self-induced irradiance, i.e.

$$
G_{a \delta>1, a \delta<1, \text { inside }}=\frac{G_{\text {self }, \text { ring }}}{G_{\text {self }, \text { sphere }}} G_{\text {sphere }}
$$

Recall that the solution outside the core of an optically-thin ring is approximated by the thin ring approximation discussed in section 4.1.
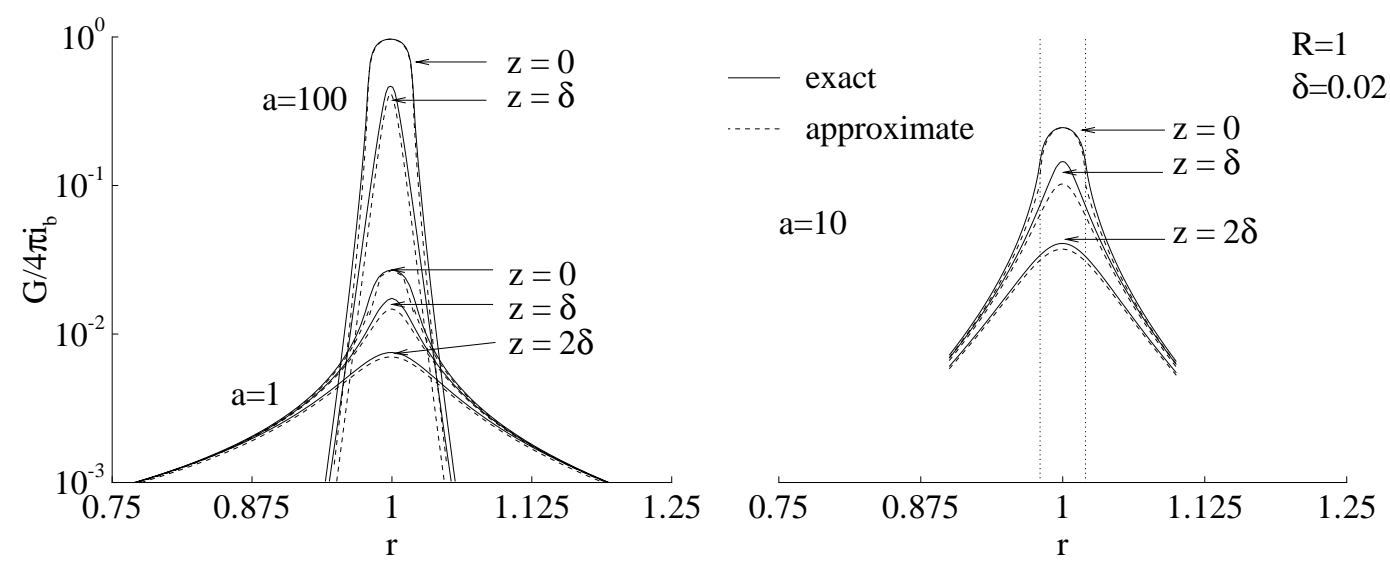

Figure 6: Exact vs. approximate solutions of $G$ for $a=1,10$ and 100.

Using the model just layed out, approximate vs. exact calculations of the radial profiles of $G$ at various elevations are presented in Fig. 6 for values of $a$ of 1,10 and 100 respectively. 


\section{Extension to Cartesian Coordinates}

In three dimensional Cartesian coordinates, the medium may be decomposed into spherical computational elements. The solution due to sphere is given by Eqs. 9 and 10. For two dimensional rectangular geometries, the computational elements are infinite circular cylinders. The irradiance due to a infinite cylinder [4] of radius $R$ and absorption coefficient $a$ at radial location $r$ is given by

$$
\begin{gathered}
\frac{G(r \leq R)}{4 \pi i_{b}}=a R \int_{1}^{\infty} \frac{I_{1}(\bar{a} y R) K_{0}(\bar{a} y r)}{y} d y \\
\frac{G(r \geq R)}{4 \pi i_{b}}=a R \int_{1}^{\infty} \frac{\rho I_{1}(\bar{a} y r) K_{0}(\bar{a} y r)+I_{0}(\bar{a} y r)\left(\rho K_{1}(\bar{a} y r)-K_{1}(\bar{a} y R)\right)}{y} d y
\end{gathered}
$$

where $I$ and $K$ are the modified Bessel functions of first and second kind respectively, $\rho \equiv \frac{r}{R}$, and $a$ is the "effective attenuation coefficient" given by Eq. 8 .

\section{Example I}

To validate our model and check its accuracy, we use the following example in which the absorption coefficient is assumed to be constant throughout the domain.

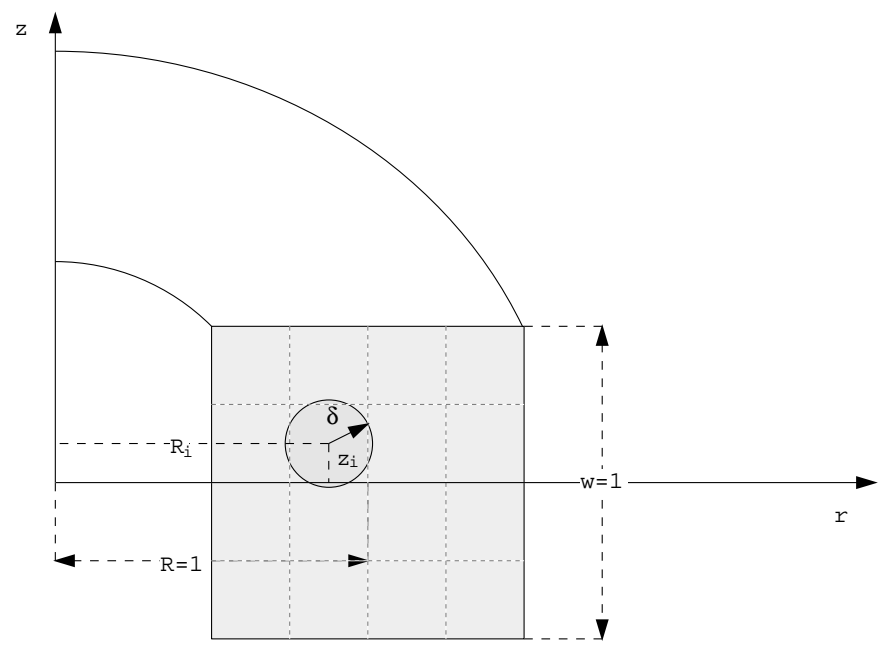

Figure 7: Schematic for example I.

We consider a ring with square cross section, as shown in Fig. 7, with radius $R=1$ and side $w=1$. The objective is to compare the irradiance calculated using the model with that computed using exact numerical integration. The comparison is done in terms of radial profiles of $G$ passing through the center of the domain for three values of the absorption coefficient: $a=1,10$, and 100 .

The mesh used to perform the numerical integration was tested for each case to obtain a mesh independent solution. As expected from the nature of the kernel, the number of points in each dimension required for an accurate solution is proportional to $a$, the reason being the steeper gradients due to the exponential term. For $a=1$, for example, we use a $100 \times 100 \times 100$ mesh. To cut down the cost, we used a cutoff distance to alleviate computing the contribution of those points of the domain that are at a distance $d$ from the target point such that $a d=6$.

Implementation of our model demands discretizing the domain into $N$ computational element that are essentially rings characterized by a radius $R_{i}$ and core radius $\delta_{i}, i=1, N$. The rings are constructed by discretizing the domain using a rectangular mesh, and representing each cell by a ring located at the center of the cell with a core radius selected such that the 
thermal energy of the cell is conserved. We are interested in values of $\delta$ that are typical to a those used in flow and/or combustion calculations. We choose $\delta_{i}=0.02$, i.e. we use $50 \times 50$ rings for all the cases (independent of $a$ ). The use of cutoff distance is also implemented in these computations.

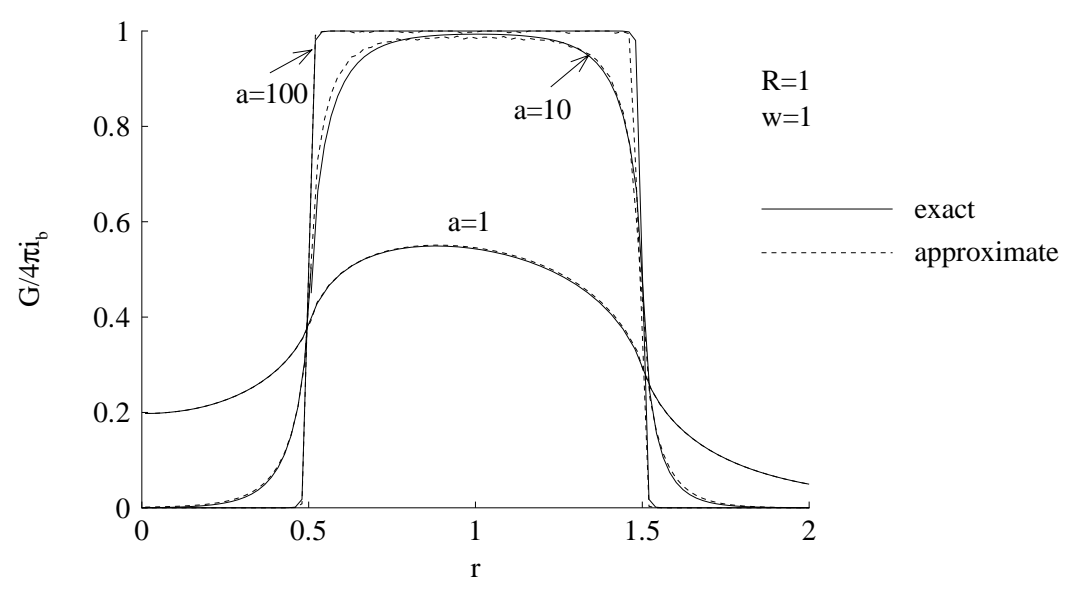

Figure 8: Exact vs. approximate solutions of $G$ for $a=1,10$ and 100.

The results are presented in Fig. 8 where the irradiance normalized by the black body intensity is plotted versus the radial location $r$. For the case of $a=1$, the value of $G$ on the $z$-axis is non-zero which is due to low attenuation $(a=1)$ experienced by the intensity in a certain direction. Due to the same reason, the maximum value of $G$ occurs on the side of the domain that is closer to the axis, where the azimuthal distribution of the domain has a significant contribution. This would not be the case for higher values of $a$, as seen in Fig. 8. In this case, the domain is optically thick and the attenutation is large leading to a fast decay in the vicinity of the domain. This explains the sharp rise (drop) in the value of $G$ around $r=0.5$ (1.5). The behavior is even more obvious in the case of the optically thick limit where $a=100$. In this case, the irradiance has a top-hat profile, where no intensity escapes the domain. Obviously, for the three cases, the model compares satisfactorily with the exact solution.

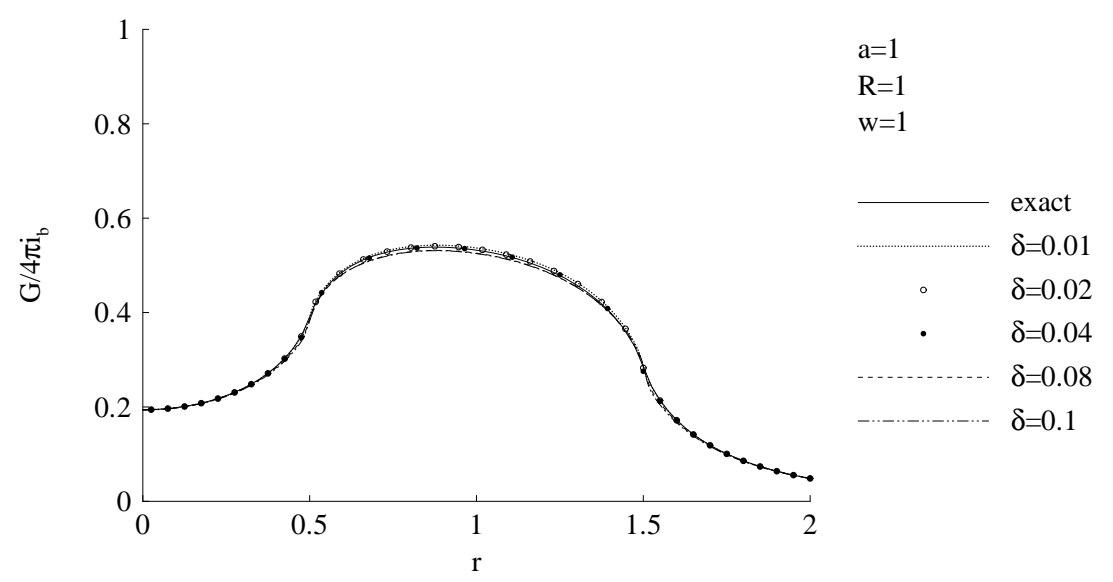

Figure 9: Exact vs. approximate solutions of $G$ for $a=1, \delta=0.01,0.02,0.04,0.08,0.1$. 


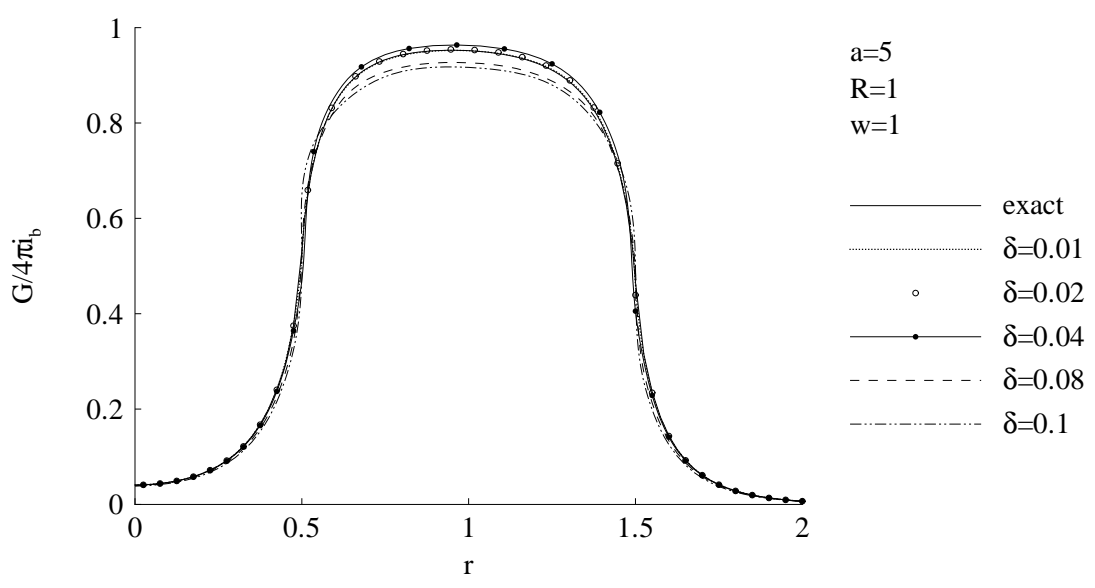

Figure 10: Exact vs. approximate solution of $G$ for $a=5, \delta=0.01,0.02,0.04,0.08,0.1$.

\subsection{Convergence}

The accuracy of the discrete source method depends on the "optical core radius" of the computational element, i.e. $\bar{a} \delta$. According to the model, accuracy is expected to improve as $\bar{a} \delta$ departs from unity, i.e. $\bar{a} \delta \ll 1$ or $\bar{a} \delta \gg 1$. On the other hand accuracy is expected to be least when $\bar{a} \delta \sim 1$. To prove that the model behaves as expected, we solve for the irradiance induced by the isothermal ring of square cross section, see Fig. 7 for the schematic. The results are presented is in Figs. 9 and 10, corresponding to values of $a$ of 1 and 5 respectively. In Fig. 9 , where $a=1$, radial profiles of the irradiance at $z=0$ are plotted for different core sizes of the computational elements. Numerical results shows a satisfactory agreement with the exact solution for all selected values of $\delta$. This behavior is expected since $a \delta \ll 1$ for all selected core sizes. Departure of the numerical solution from the exact solution, however, shows in Fig. 10, where $a=5$, as the core size increases $(\delta=0.08,0.1$ for example). In this case, the optical core radius $a \delta$ is of the order of unity $(a \delta=0.4,0.5)$. Convergence to the exact solution occurs as $\delta$ decreases, which corresponds to $a \delta \ll 1$.

\section{$9 \quad$ Example II}

Even though the example presented above provides the validation required for the suggested model, it was based on the assumption that the absorption coefficient is uniform in space and that the volume of gas considered has a uniform temperature that is higher than that of the cold surrounding. A more realistic example would obviously be that of a medium associated with a temperature distribution within and correspondingly variable absorption coefficient. Here we consider methane fuel ring, Fig. 11, that is reacting with the atmosphere.

The objective is to determine the radiation source distribution. The reaction is assumed to be single-step and to proceed at an infinite rate: $\mathrm{CH}_{4}+2\left(\mathrm{O}_{2}+3.76 \mathrm{~N}_{2}\right) \longrightarrow \mathrm{CO}_{2}+2 \mathrm{H}_{2} \mathrm{O}+$ $7.52 N_{2}$. Based on a one dimensional model, the corresponding species distributions are shown in Fig. 12 .

The absorption coefficient is obtained according to the following equation [1]:

$$
a(T)=\sum_{f, o, p, d} a_{p, i}(T) p_{i}
$$

where $i$ corresponds to a species, and $a_{p, i}$ is the Plank-mean absorption coefficient of species $i$, and $p_{i}$ is the partial pressure of the species. The Plank-mean absorption coefficient is defined as 


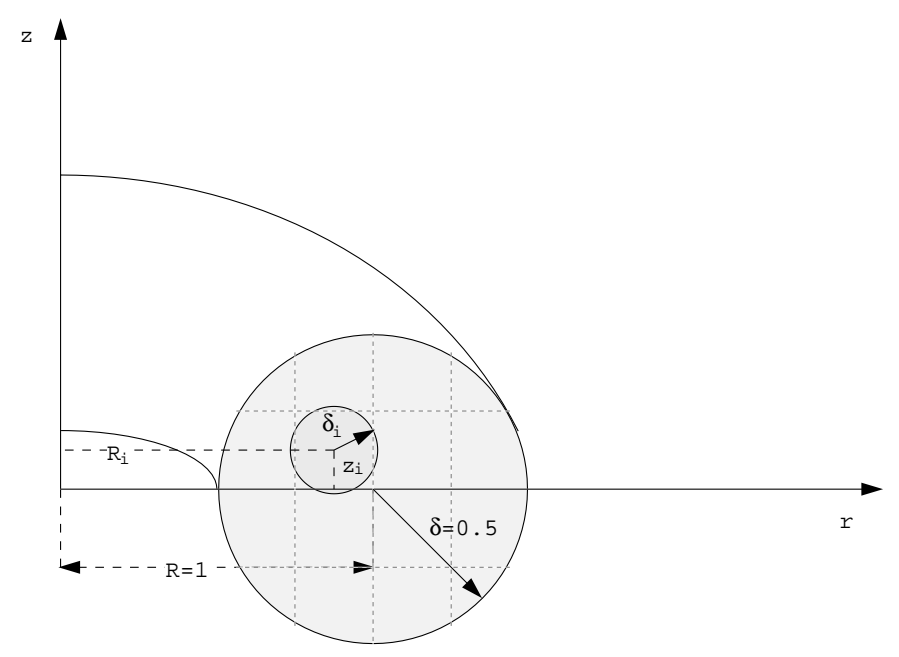

Figure 11: Schematic for example II.

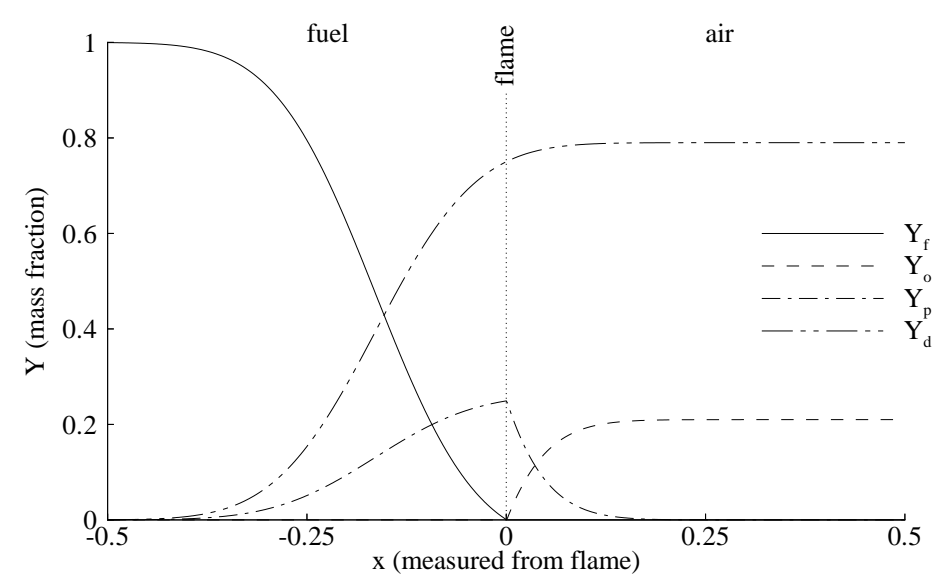

Figure 12: Species distributions of 1-D diffusion flame.

$$
a \equiv \frac{\int_{0}^{\infty} i_{b \eta} a_{\eta} d \eta}{\int_{0}^{\infty} i_{b \eta} d \eta}
$$

where $\eta$ is the wavenumber, $i_{b \eta}$ is the Plank function, and $a_{\eta}$ is the spectral absorption coefficient.

The temperature dependence of $a_{p}$ for the various species, obtained using narrow band averaged values for the absorption coefficient (tien), is presented in Fig. 13. The absorption coefficient of air is negligible for the range of temperature we are interested in.

The temperature distribution and the corresponding absorption coefficient distribution for the 1-D flame are shown in Fig. 14.

The temperature and absorption coefficient distributions we select for our example are presented in Fig. 15. These distributions are based on the 1-D distributions.

The exact solution for the divergence of the heat flux is based on direct numerical integration of Eq. 5. The numerical parameters were selected along the same lines discussed in Example I. In obtaining the discrete source method solution, three sizes of computational elements were used: $\delta=0.01,0.02$ and 0.04 . As mentioned before, the effective attenutation coefficient was obtained using Eq. 8. The results are presented in Fig. 16. The solutions obtained using the discrete source method agree well with the exact solution for the element sizes used. The slight 


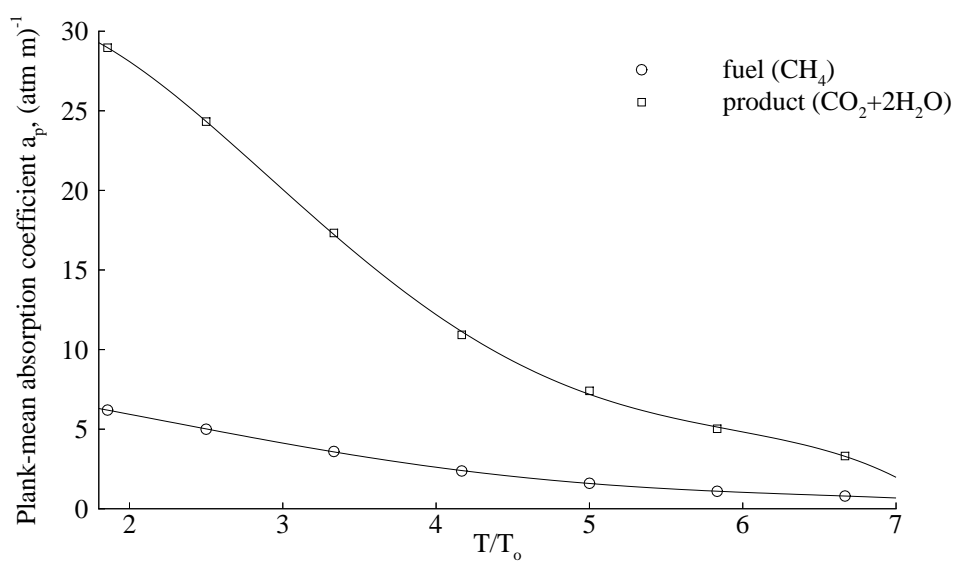

Figure 13: Dependence of the Plank-mean absorption coeffcient on temperature [5]

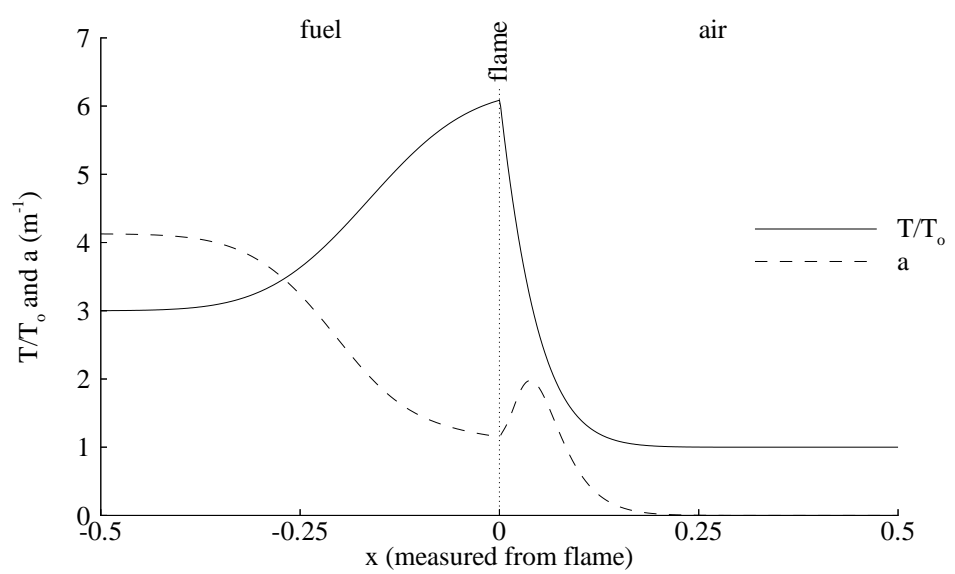

Figure 14: Temperature and absorption coefficient distribution of the 1-D flame.

deviation for the case of $\delta=0.04$ is due to assumption involved in evaluating the effective attenuation coefficient, i.e. when evaluating the inner integral of Eq. 5, we assume that the source element is represented by its center $\left(s^{\prime}=0\right)$.

\section{Conclusion}

A grid-free Lagrangian scheme for calculating the radiative source term in the energy equation has been presented. The Discrete Source Method is compatible with the vortex and transport element methods. The solution is presented in terms of "Biot-Savart" summation over a collection of elements discretizing the domain. The field induced by each element is expressed in two forms depending on the "optical thickness" of the element, and the fields can be obtained from analytical expressions or a tabulated integral in two dimensions. In three dimensions, only analytical expressions are used, Eqs. (9) and (10). An attractive feature of the method is the use of the concept of "effective attenuation coefficient" which proved to be a useful tool of analysis. Lagrangian schemes are based on following a collection of elements in time, which may be thought of as moving radiation volumes. In such a case, methods based on precalculation of the 


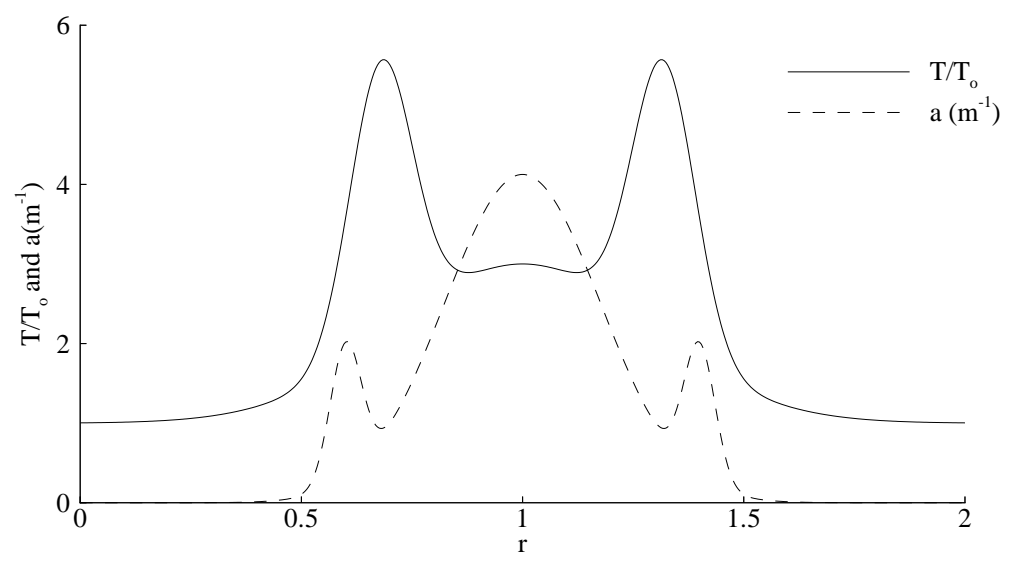

Figure 15: Temperature and absorption coefficient distributions used in Example II.

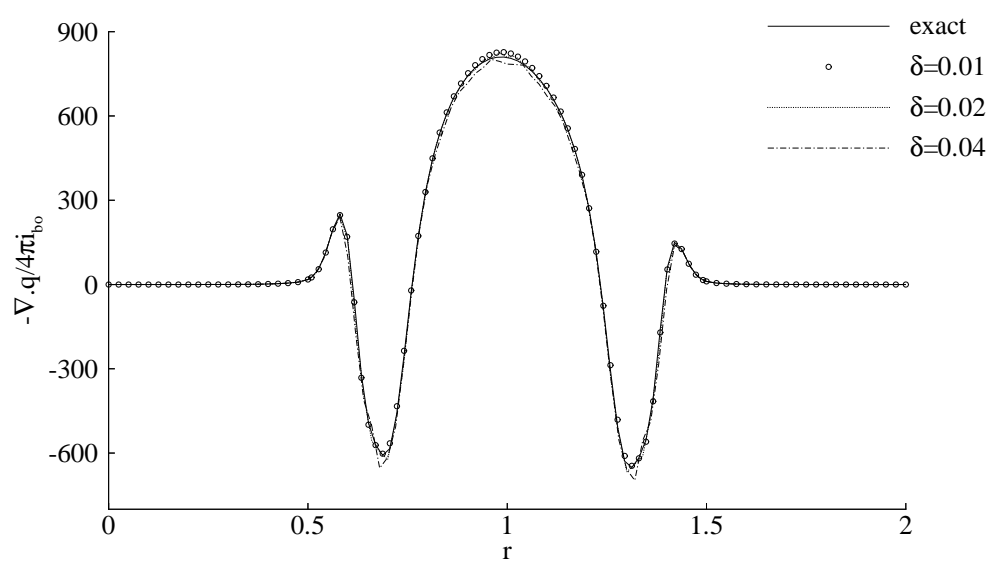

Figure 16: Radiation source distribution for Example II.

exchange factors for a particular geomtry, such as the zonal method [6], lose their advantage. For moving elements, the exchange factors has to be evaluated every time step. Comparison between the Discrete Source Method and other numerical method will be discussed in a future publication.

\section{References}

1 Modest, M.: Radiative Heat Transfer, McGraw Hill, Inc. (1993).

2 Tan, Z.: "Radiative Heat Transfer in Multidimensional Emitting, Absorbing, and Anisotropic Scattering Media - Mathematical Formulation and Numerical Method", Journal of Heat Transfer, vol. 111, (1989).

3 Thynell, S.T. and Ozisik, M.N.: "Radiation Transfer in an Isotropically Scattering Homogeneous Solid Sphere", J. Quant. Spectrosc. Radiat. Transfer, vol. 33, No. 4, pp. 319-330, (1985).

4 Heaslet, M.A. and Warming, R.F.: "Theoretical Predictions of Radiative Transfer in a Homogeneous Cylindrical Medium", J. Quant. Spectrosc. Radiat. Transfer, vol. 6, pp. 751-774, (1966). 
5 Tien, C.L.: "Thermal Radiation Properties of Gases", Advances in Heat Transfer, vol. 5, Academic Press, New York, pp. 253-324, (1968).

6 Hottel, H.C. and Sarofim, A.F.:Radiative Transfer, McGraw-Hill, New York, 1967. 